\title{
Uso de sondeos electromagnéticos en la caracterización hidrológica del acuífero del altiplano de Tula, Tamaulipas
}

\author{
The use of electromagnetic soundings for the hydrological \\ characterization of the aquifer of the highlands of Tula, Tamaulipas
}

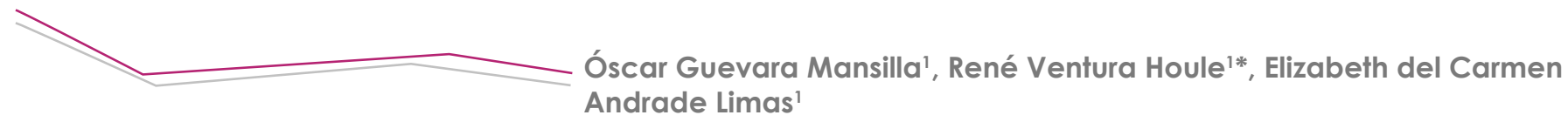

Guevara Mansilla, O., Ventura Houle, R., Andrade Limas, E. C. Uso de sondeos electromagnéticos en la caracterización hidrológica del acuífero del altiplano de Tula, Tamaulipas. Investigación y Ciencia de la Universidad Autónoma de Aguascalientes. Número 70: 23-30, enero-abril 2017.

\section{RESUMEN}

Las técnicas de exploración mediante sondeos electromagnéticos consisten en transmitir un campo magnético, el cual viaja a través del subsuelo, la disminución del campo electromagnético está asociada a la resistividad de la litología presente, lo que permite identificar materiales con posibilidades de contener agua, así como el volumen de los mismos. Esto en conjunto con variables hidrogeológicas funciona como gran apoyo al momento de gestionar adecuadamente el uso del agua subterránea. Se realizaron 40 sondeos en el altiplano de Tula, Tamaulipas, región cuya única fuente hídrica viable es el agua subterránea. Se identificaron tres unidades hidrogeológicas, en las cuales se estableció una zona sin capacidad para almacenarla, otra con baja capacidad para el mismo objetivo y la tercera, que es una unidad de transporte, lo que indica que los afloramientos de la formación geológica El Abra son la zonas de recarga del sistema acuífero.

\section{ABSTRACT}

The time domain electromagnetic surveys consist of transmitting an electric field, which travels through

Palabras clave: sondeos electromagnéticos, hidrogeología, gestión de agua, geofísica, acuífero.

Keywords: electromagnetic soundings, hydrogeology, water management, geophysics, aquifer.

Recibido: 10 de agosto de 2015, aceptado: 21 de julio de 2016

Facultad de Ingeniería y Ciencias, Universidad Autónoma de

Tamaulipas, México.

* Autor para correspondencia: rventura@uat.edu.mx geological materials whose resistivity such emition as lithology, is measured allowing to identify materials likely to contain water and the volume thereof. This, together with hydrogeological variables, works as great support when properly managing groundwater use. Forty wells were drilled in the highlands of Tamaulipas Tula region, whose only viable water source is groundwater. Three hydrogeological units, where an area was identified without water storage capacity, another with low capacity to hold water and the third as a unit of transportation, indicating that the outcrops of the geological formation El Abra, are the recharge areas of the aquifer system.

\section{INTRODUCCIÓN}

Una de las técnicas geofísicas con mayor aplicación en la exploración del agua subterránea son los sondeos electromagnéticos en el dominio del tiempo (TDEM, por sus siglas en inglés). Estos consisten en hacer circular un campo eléctrico alrededor de una bobina transmisora en periodos de tiempo de milisegundos. Durante el periodo de conexión se establece un campo magnético estable en el subsuelo, y cuando este se corta de forma instantánea, el campo electromagnético causa corrientes parásitas que se propagan en el terreno. Como consecuencia de pérdidas de resistencia calórica estas corrientes disminuyen con el tiempo provocando un campo magnético decreciente en la superficie, el cual puede ser medido. Cuando en el subsuelo hay cuerpos de conductividad eléctrica elevada, la atenuación de las corrientes parásitas es significativamente menor que en materiales dieléctricos; por tanto, la medida de la relación de decrecimiento del campo secundario proporciona 
IIVESTIGACIÓn Y CIERCIA DE LA UחIVERSIDAD AUTÓNOTH

24 DE RGUASCALIERTES una forma de detectar la presencia de cuerpos conductores en el subsuelo, así como estimar su conductividad y resistividad (Casas, 2000).

Es de esta forma que los TDEM muestran la distribución del subsuelo en términos de homogeneidad, basados en la caracterización resistiva. En las secciones del subsuelo ha sido posible observar las heterogeneidades debidas a estructuras geológicas, cambios de facies y fracturación de la roca, donde además la presencia del agua provoca cambios importantes en la resistividad eléctrica (Navarro Luna, 2006). Los TDEM han sido utilizados en Omán y México por Young et al. (1998) y Ramos Leal et al. (2007) con el propósito de definir la geometría de los acuíferos; en Grecia por Kanta et al. (2013), para conocer las características hidrogeológicas del acuífero; en Guatemala, donde Lepper y Curtis (2005) utilizaron los TDEM para localizar agua subterránea; en Arabia Saudita y Egipto, Al Garni y El Kaliouby (2011) y Khalil et al. (2013) los utilizaron para el mapeo de la intrusión marina. Todos estos trabajos con el fin de identificar áreas óptimas para la extracción de agua subterránea.

La zona acuífera del altiplano de Tula se encuentra en una planicie intermontana de la Sierra Madre Oriental, al suroeste del estado de Tamaulipas, México, y forma parte del acuífero Tula-Bustamante, que corresponde a la Región Hidrológica No. 37, El Salado (CONAGUA, 2002). La zona presenta un clima semiárido con precipitaciones anuales de $400 \mathrm{~mm}$ y temperaturas que varían durante el año desde $0{ }^{\circ} \mathrm{C}$ hasta $40{ }^{\circ} \mathrm{C}$ (Gobierno del estado de Tamaulipas, 2011 ). A causa de las bajas precipitaciones, los cuerpos de agua superficiales son escasos e intermitentes, lo que hace del acuífero del altiplano de Tula el recurso hídrico más importante para 27,500 personas que habitan en la región, y que tienen como principal actividad económica la agricultura y ganadería (INEGI, 2011). De acuerdo con el Servicio de Información Agroalimentaria y Pesquera (s. f.), el incremento de la superficie sembrada bajo condiciones de riego de 735 ha (2003) a 3,238 ha (2013), causó un aumento en el consumo de agua subterránea. Debido al alto impacto socioeconómico que tiene el acuífero del altiplano de Tula, es importante conocer su geometría, los materiales geológicos que lo integran y sus propiedades hidráulicas, con la finalidad de entender el funcionamiento e identificar las zonas de recarga y descarga de agua subterránea, así como áreas óptimas para la perforación de pozos de agua.

Plata Torres (2000) menciona que las técnicas de prospección geofísica tienen un amplio rango de aplicaciones en la caracterización del agua subterránea, que van desde la definición de la geometría de los acuíferos hasta la determinación de las características hidráulicas relacionadas con la litología. Esto ha permitido la identificación de las unidades hidrogeológicas (UH) que conforman un acuífero, definidas por Sánchez San Román (2014) como el conjunto de formaciones geológicas con un funcionamiento hidráulico similar. Es por lo anterior que el presente trabajo tuvo como objetivo determinar las unidades hidrogeológicas que integran el acuífero del altiplano de Tula, esto mediante la recopilación de información de 40 TDEM, los niveles piezométricos de 12 pozos de extracción de agua subterránea e información hidrogeológica y estratigráfica.

\section{MATERIALES Y MÉTODOS}

\section{Descripción del área de estudio}

El altiplano de Tula es una planicie intermontana que se encuentra al poniente de la Sierra Madre Oriental en el municipio de Tula, Tamaulipas (Figura 1). Esta

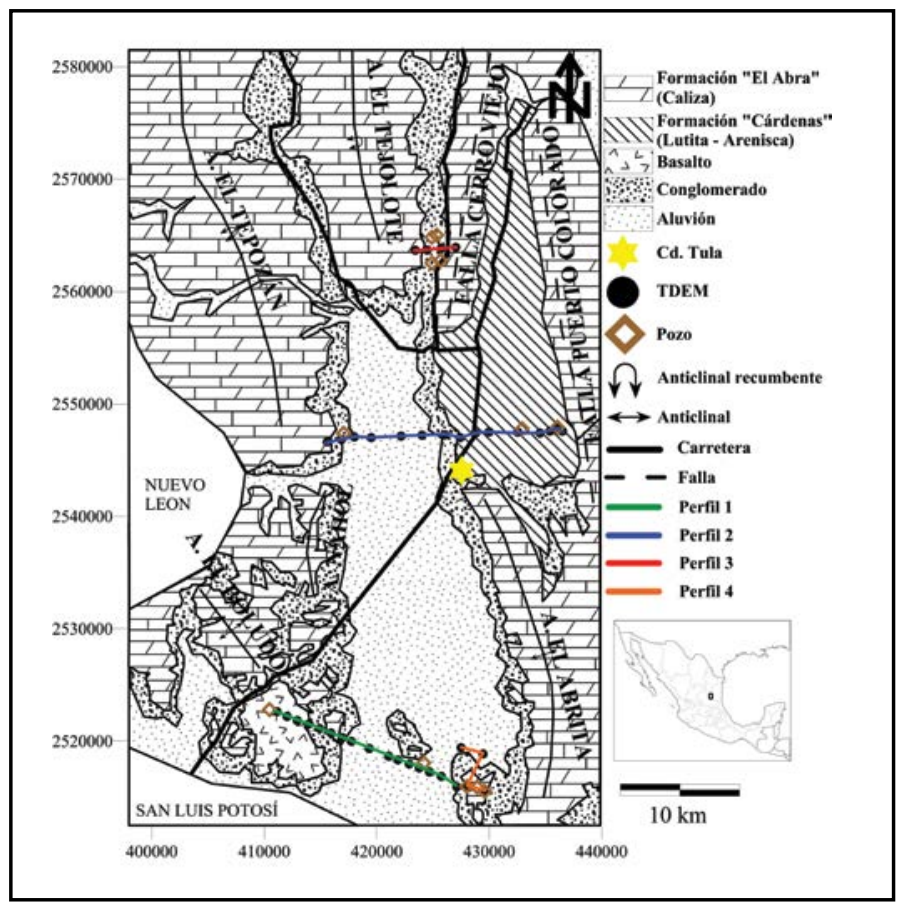

Figura 1. Geología de la zona de estudio, ubicación de los TDEM y pozos de interés. 


\section{IIVESTIGAGIÓn Y CUERCIA DE LA UกIVERSIDAD AUTÓOOOMA DE RGUASCALIERTES}

región se encuentra constituida por afloramientos de diversos materiales geológicos que, debido a sus propiedades litológicas similares, son descritas como formaciones.

La formación más antigua que aflora en la zona de estudio es El Abra, del Cretácico Inferior, esta emerge para formar los anticlinales El Abrita, El Tepozán, El Tejolote, Nahol, El Boludo; así como las fallas Cerro Viejo y Puerto Colorado (SGM, 2006). Dicha formación es descrita por Carrillo (1961) como una plataforma de rocas sedimentarias arrecifales, como son calcilutitas, calcarenitas y conglomerados calcáreos, masivos, de color crema ligeramente grisácea, con abundantes rudistas y fragmentos de otros macro y microfósiles. También aflora la formación Cárdenas (Tabla 1), que se encuentra constituida por una sucesión de 1,800 $\mathrm{m}$ de espesor de siliciclásticos finos con capas de calizas intercaladas que se depositaron en aguas someras en la plataforma Valles-San Luis Potosí a finales del Cretácico Superior (Oviedo García, 2005). La formación es descrita y dividida por Myers (1968) en tres miembros: inferior, medio y superior. En el miembro inferior predominan las calizas y también se presentan estratos de arcilla, arenisca y bioesparudita; el miembro medio está compuesto por intercalaciones de lutita, arenisca y caliza; y el miembro superior por areniscas, lutitas, caliza y marcas de ondulitas (también conocidas como ripples, su nombre en inglés). A las faldas de los anticlinales se encuentra material Conglomerado Reynosa (SGM, 2006), del periodo Terciario, constituido por sedimentos continentales con granulometrías que varían de arcillas a gravas; estos son fragmentos de calizas, areniscas, pedernal y rocas ígneas que se engloban en una matriz arcillosa y cementados por carbonatos, sus espesores varían desde 10 hasta $30 \mathrm{~m}$ (SGM, 2008). Además, al suroeste aflora una brecha volcánica de los periodos TerciarioCuaternario, dicha grieta se compone de basaltos de olivino vesiculares de textura holocristalina color gris obscuro (SGM, 2008). Por último se encuentra material aluvial del Cuaternario formando una llanura, este se presenta como clastos de granulometría que van desde tamaño arcilla hasta cantos rodados de $20 \mathrm{~cm}$, producto de la desintegración de rocas preexistentes, principalmente calizas y lutitas (SGM, 2008).

\section{Sondeos electromagnéticos en el dominio del tiempo (TDEM)}

En el área de estudio se hicieron 40 TDEM con el equipo terraTEM (Figura 2), en cada punto se hicieron cinco mediciones, las cuales operaron con 8 A y un número creciente de ventanas en cada corrida, que fue de: 28 , 30, 32, 34 y 36. La información obtenida fue procesada con el programa computacional WinGlink. Los TDEM se hicieron para abarcar todos los materiales geológicos superficiales de la zona, como se muestra en la Figura 1. A partir de los sondeos se formaron tres perfiles geoeléctricos con longitudes de 19, 23 y $3.8 \mathrm{~km}$ con $800 \mathrm{~m}$ de profundidad cada uno, y otro perfil con longitud de $9 \mathrm{~km}$ y $500 \mathrm{~m}$ de profundidad; el tamaño de la bobina utilizada para llegar a una profundidad de $800 \mathrm{~m}$ fue de 225 por $225 \mathrm{~m}$, y para alcanzar la profundidad de $500 \mathrm{~m}$ se utilizó una bobina de 150 por $150 \mathrm{~m}$, ambas bobinas con configuración loop coincidente.

Tabla 1. Estratigrafía del municipio de Tula (SGM, 2006)

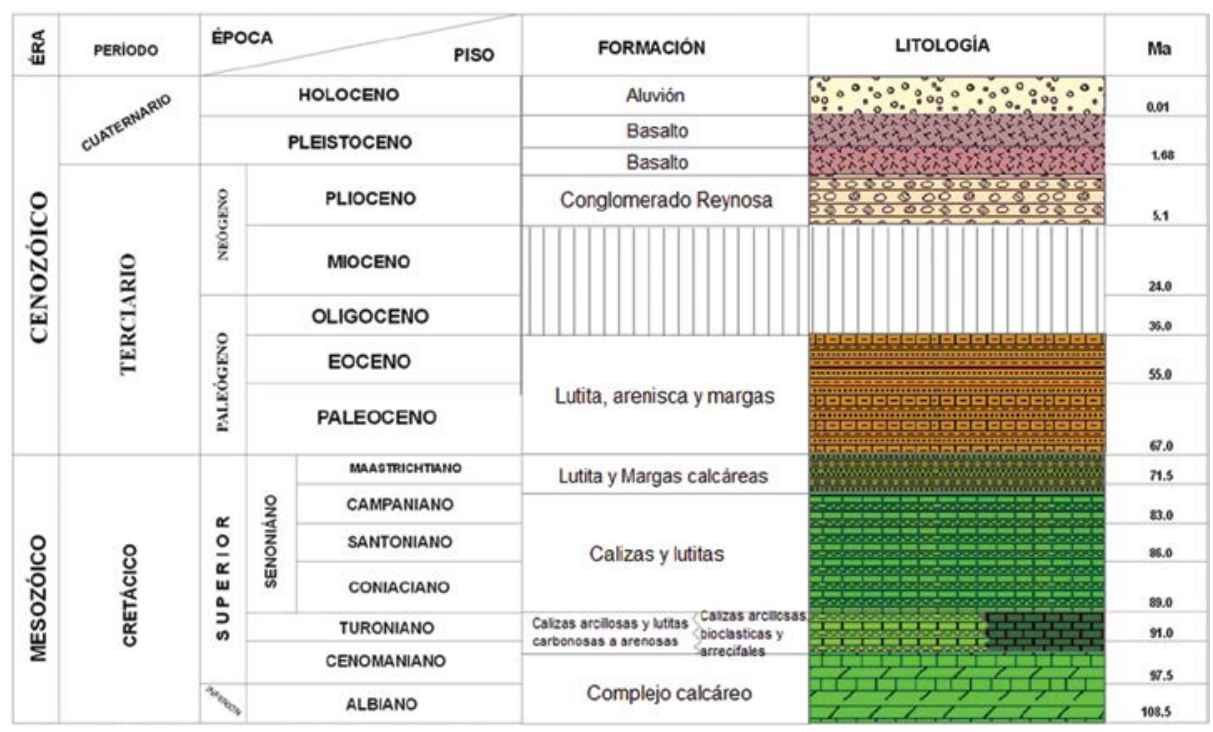




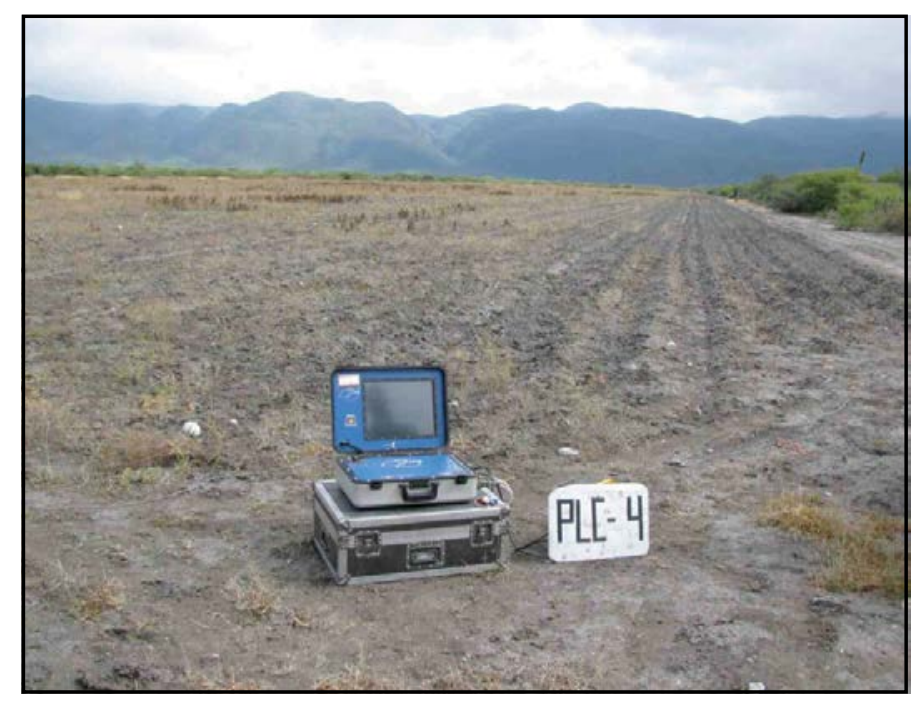

Figura 2. Sondeo electromagnético en el dominio del tiempo realizado en el altiplano de Tula, con el equipo terraTEM y arreglo loop coincidente. Fotografía de René Ventura Houle.

\section{Piezometría}

Se midió el nivel piezométrico de 12 pozos de agua que se encontraban cerca de los perfiles geoeléctricos (Figura 1) para relacionarlos con sus valores resistivos, ya que según Navarro Luna (2006) la presencia de agua provoca cambios importantes en la resistividad eléctrica. Los niveles piezométricos fueron medidos con la sonda Solinist $102(150 \mathrm{~m}$ de longitud). El procedimiento consistió en hacer bajar la sonda a través de los pozos de extracción de agua, como tiene carga eléctrica, al entrar en contacto con el agua completa un circuito que genera una alarma, la cual indica que se detenga el descenso de la sonda. Así se tomó la lectura del nivel piezométrico.

\section{Correlación de la información obtenida}

Se interpretó cada perfil geoeléctrico, se relacionaron los valores resistivos que los mismos presentan con los valores de resistividad comunes de algunos materiales geológicos que describen Goldman y Neubaver (1994) y se correlacionó esto con la geología superficial (SGM, 2006), como lo hicieron Aldama et al. (2002) y Cruz Falcón (2007).

\section{RESULTADOS}

Con base en la información recabada, el acuífero del altiplano de Tula se divide en cuatro unidades georresistivas definidas por el rango de resistividad eléctrica que presenta el subsuelo (Tabla 2).

Tabla 2. Unidades geoeléctricas y su posible correlación de acuerdo con su intervalo georresistivo

\begin{tabular}{|c|c|l|}
\hline $\begin{array}{c}\text { Unidad } \\
\text { geoeléctrica }\end{array}$ & $\begin{array}{c}\text { Intervalo } \\
\text { georresistivo } \\
\text { (Ohm.m) }\end{array}$ & Posible correlación \\
\hline U1 & $150-300$ & Se relaciona con calizas arcillosas y lutitas calcáreas. \\
\hline U2a & $5-30$ & Unidad que se asocia con materiales geológicos que almacenan agua. \\
\hline U2b & $30-150$ & $\begin{array}{l}\text { Se interpreta como rocas ígneas máficas fracturadas (basalto), o también se reconoce } \\
\text { como rocas sedimentarias detríticas (lutitas y areniscas). }\end{array}$ \\
\hline U3 & $300-6000$ & Corresponde a materiales calcáreos como calizas y dolomías o rocas ígneas basálticas. \\
\hline
\end{tabular}

Elaboración propia.

\section{DISCUSIÓN}

\section{Perfil 1}

Tiene una distancia de 20 km (Figura 1) con dirección este-oeste y una profundidad de $800 \mathrm{~m}$ (Figura 3: a y b). A la izquierda de este perfil, entre los kilómetros 0 y 10, emerge a la superficie una brecha volcánica, que se corrobora con la geología superficial debido a que en la zona se observan rocas basálticas (SGM, 2006). Estas se encuentran representadas por las unidades U3, U2b y U2a, las cuales se identifican como basalto, basalto fracturado y basalto saturado de agua, respectivamente; este último se confirma por los valores resistivos a la profundidad del nivel piezométrico de un pozo de extracción de agua ubicado sobre dicho material (Figuras ly 3: a y b).

La unidad georresistiva U2a a la derecha del perfil 1 (Figura 3a) es identificada por la geología superficial como material aluvial (SGM, 2006), este es producto de la desintegración de las rocas circundantes (SGM, 2008). A pesar de que el material aluvial presenta una 


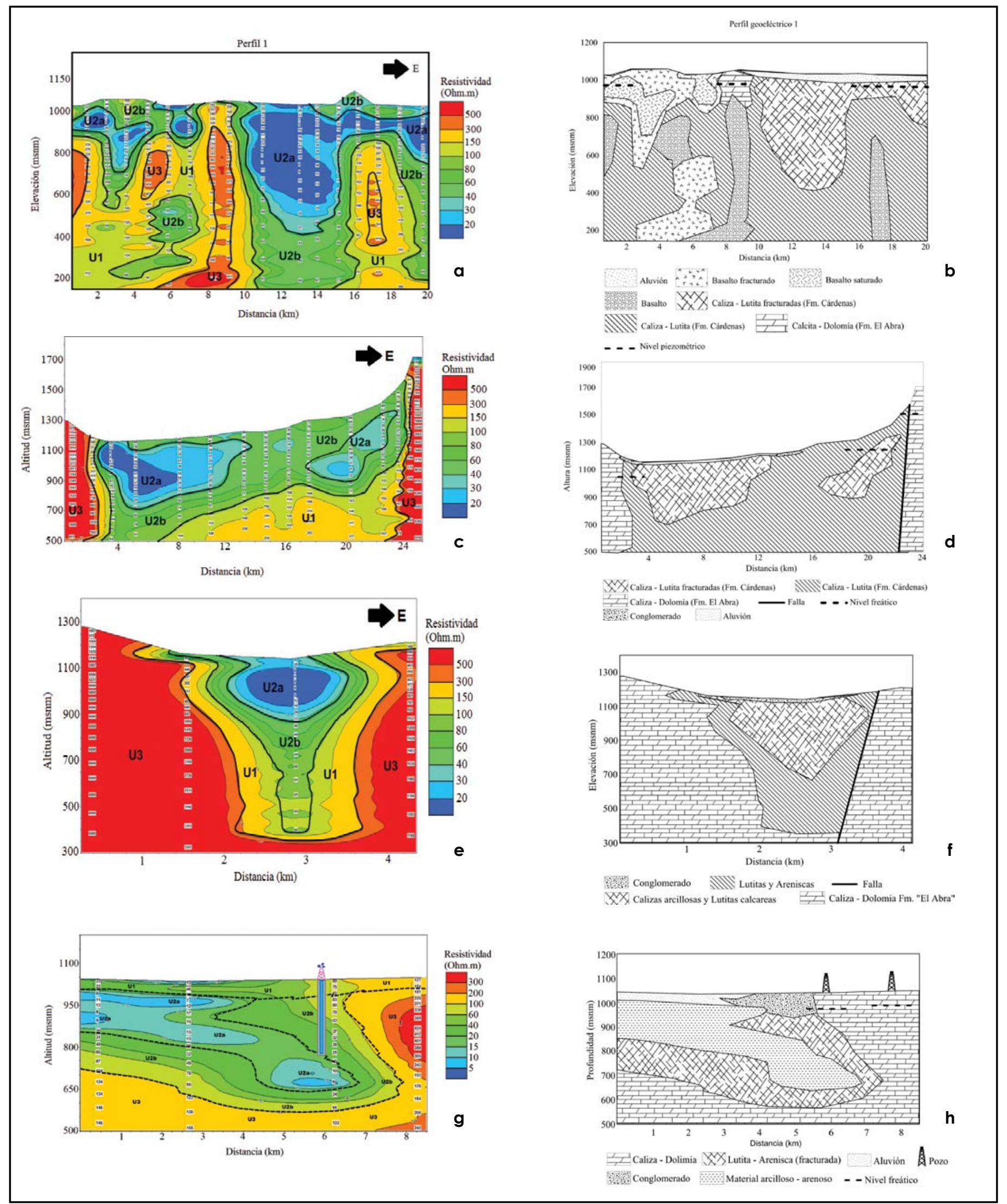

Figura 3. Perfiles geoeléctricos e interpretación de materiales representados. 
IIVESTIGACIÓn Y CIERCIA DE LA UNIVERSIDAD AUTÓNOMA

resistividad baja debido a la presencia de agua, no está saturado, ya que el nivel freático se encuentra a $69.5 \mathrm{~m}$ de profundidad en los pozos cercanos al perfil, cuando el espesor del material aluvial es de $30 \mathrm{~m}$ y se localiza en la superficie (SGM, 2008); esto indica que el material aluvial sirve como zona de recarga vertical del acuífero.

Por debajo de la unidad U2a descrita anteriormente se encuentran las unidades U2b y U1, las mismas se identifican como la formación Cárdenas; sin embargo, presentan rangos de resistividad mayores debido a la ausencia de fracturas y agua. Esta formación se ve deformada a la derecha por un intrusivo de roca volcánica representada por la unidad U3.

\section{Perfil 2}

Las Figuras 3: c y d representan una longitud de $24 \mathrm{~km}$ con dirección este-oeste y una profundidad de $800 \mathrm{~m}$. Las unidades U3 a los extremos izquierdo y derecho del perfil 2 se encuentran compuestas por rocas calizas y dolomías, donde según la estratigrafía y la geología estructural (SGM, 2006, 2008) son pertenecientes a la formación El Abra del Cretácico Inferior, la cual aflora a la izquierda en el anticlinal Tepozán y a la derecha en la falla Puerto Colorado.

Las unidades U2a, U2b y U1 del perfil 2 (Figura 3c) representan a la formación Cárdenas, donde U2a se refiere a materiales saturados de agua, lo que se demuestra por la extracción de agua a 149 m de profundidad de un pozo cercano, y las unidades U2b y UI presentan un mayor rango de resistividad debido a que se encuentran ausentes de fracturas y agua, 0 bien presentan una mayor compactación (Juanah et al., 2013). La formación Cárdenas aflora a la derecha del perfil como la unidad U2b y va descendiendo a la izquierda para ser cubierta por una capa de material aluvial y conglomerado (SGM, 2006).

La unidad U2a a la izquierda del perfil 2 (Figura 3c) tiene un espesor aproximado de $300 \mathrm{~m}$, lo que le permite ser recargada de agua por sistemas de flujo locales, intermedios y regionales, los cuales se presentan a diferentes profundidades (Tóth, 1999) y tienen una composición química correspondiente al tiempo de su recorrido que va de acuerdo con la secuencia de Chevotareb (Sánchez, 2012); esto es comprobable por la presencia de agua de facies mixtas en la zona reportada por Saldierna (2014).
Del mismo modo, la unidad U2a a la derecha del perfil 2 (Figura 3c), con un espesor aproximado de $350 \mathrm{~m}$, se ve afectada por flujos locales, intermedios y regionales. Esta unidad se encuentra en una zona que presenta agua con una composición química Na-mixta (Saldierna, 2014), que de acuerdo con la secuencia de Chevotareb corresponde a un flujo de agua regional; sin embargo, la presencia del ion $\mathrm{Na}^{+}$se atribuye principalmente a su disolución y abundancia (Sánchez San Román, 2012), presente en la formación Cárdenas debido a su origen sedimentario en aguas someras (Oviedo García, 2005).

\section{Perfil 3}

El tercer perfil (Figura 3: e y f) se encuentra ubicado en el sinclinal Calabacillas con dirección este-oeste, una longitud de $4 \mathrm{~km}$ y profundidad de $800 \mathrm{~m}$. Las unidades georresistivas que dividen el perfil (Figura 3: e y f) adoptan la forma de los pliegues del sinclinal. El más antiguo corresponde a la unidad U3, esta unidad representa a la formación El Abra, la cual aflora a la izquierda para formar el anticlinal El Tejolote y a la derecha la falla Cerro Viejo (SGM, 2006).

Sobre la unidad U3 descansan los pliegues que conforman las unidades U1, U2b y U2a, en ese orden ascendente. Estas tres unidades se correlacionan con la formación Cárdenas, la cual se encuentra compuesta por lutitas, calizas y estratos de arcillas y areniscas (Myers, 1968); además Quilantán et al. (2008) ubica a esta formación en los núcleos de los sinclinales.

La unidad $\mathrm{U} 2 \mathrm{a}$, relacionada con materiales que contienen agua, tiene un espesor que va desde la superficie hasta los $300 \mathrm{~m}$ de profundidad; sin embargo, no se encuentra saturada de agua sino hasta los $120 \mathrm{~m}$ de profundidad, que es lo reportado por las mediciones piezométricas cercanas al perfil 3. A pesar de que la geología superficial reportada por el Servicio Geológico Mexicano (2006) muestra al sinclinal Calabacillas cubierto por conglomerado, este material solamente llega a tener espesores de hasta 30 m (SGM, 2008); por tanto, la formación Cárdenas es la responsable de almacenar agua en sus fisuras.

\section{Perfil 4}

El perfil 4 (Figura 3: $g$ y h) se localiza a las faldas del anticlinal El Abrita y tiene forma de Z, como se muestra en la Figura 1, con una longitud de $9 \mathrm{~km}$ y una 


\section{IIVESTIGAGIÓn Y CUERCIA DE LA UחIVERSIDAD AUTÓNOMA DE RGUASCALIERTES}

profundidad de $500 \mathrm{~m}$. A diferencia de los perfiles 1, 2 y 3 que se hicieron con el propósito de identificar los materiales geológicos presentes en el subsuelo, el perfil 4 se hizo con fines de exploración de agua subterránea, es por esto que la longitud y profundidad de este perfil es relativamente pequeña en comparación con los otros perfiles; sin embargo, sirvió de complementación y comparación del perfil 1.

A la derecha del perfil se encuentra un afloramiento de la formación El Abra (U3), donde hay dos pozos de agua con un nivel estático a 65 $\mathrm{m}$ de profundidad, lo que indica que la formación presenta una porosidad fisurada, esto explica su bajo valor resistivo. Como se ha mencionado anteriormente, sobre la formación El Abra descansa la Cárdenas, la cual se representa por la unidad U2b y U2a; esta última formación se interpreta como la describe Myers (1968), calizas que presentan estratos de arcilla, arenisca y bioesparudita; los cuales están cubiertos por la unidad U1, donde la geología superficial (SGM, 2006) indica la presencia de material aluvial y conglomerado.

La unidad U2a del perfil 4 (Figura $3 g$ ) tiene un espesor aproximado de $350 \mathrm{~m}$, y al igual que el perfil
2 , se encuentra en una zona donde el agua presenta una composición química mixta-mixta debido a la presencia de flujos de agua locales, intermedios y regionales (Saldierna, 2014).

CONCLUSIONES

Los sondeos electromagnéticos en el dominio del tiempo indican que el agua es almacenada en las fisuras de las rocas pertenecientes a la formación Cárdenas, la cual tiene como basamento a la formación El Abra. La misma, además de servir como basamento a la Cárdenas, tiene la capacidad de almacenar y transmitir agua como fue discutido en los perfiles 2 y 4; por tanto, probablemente los afloramientos de la formación El Abra recargan horizontalmente el acuífero, además de que el basamento del acuífero es aún desconocido a los $800 \mathrm{~m}$ de profundidad. En el área de estudio se identificaron tres unidades hidrogeológicas: las rocas basálticas fracturadas del perfil 1; las calizas y dolomías fisuradas de la formación El Abra; y las lutitas, areniscas y calizas de la formación Cárdenas; esta última se encuentra distribuida en todo el altiplano de Tula.

LITERATURA CITADA

- AldamA, A. A. et al. Estudio hidrogeológico de los acuíferos El Hundido y Cuatro Ciénegas, Coah. Reporte técnico. México: Secretaría de Medio Ambiente y Recursos Naturales, 292 pp., 2002.

- Al GARNI, M. A. y El KALIOUBY, H. M. Delineation of saline groundwater and sea water intrusion zones using transient electromagnetic (TEM) method, Wadi Thuwal area, Saudi Arabia. Arabian Journal of Geosciences, 4(3): 655-668, 2011.

- CARRILLO, B. J. Geología del Anticlinorio Huizachal Peregrina al noroeste de Ciudad Victoria, Tamps. Boletín de la Asociación Mexicana de Geólogos Petroleros, 13, 1-98, 1961.

- CASAS, A. Prospección geofísica aplicada a la hidrogeología: Metodología y técnicas emergentes. En M. Olmo Alarcón y J. A. López Geta (Eds.), Actualidad de las técnicas geofísicas aplicadas en hidrogeología (pp. 33-41). España: Instituto Tecnológico Geominero de España, 2000.

- CONAGUA (COMISIÓN NACIONAL DEL AGUA). Determinación de la disponibilidad de agua en el acuífero Tula-Bustamante Estado de Tamaulipas. Diario Oficial de la Federación, 30 de abril de 2002.
- CRUZ fAlCÓN, A. Caracterización y diagnóstico del Acuífero de La Paz, B. C. S., mediante estudios geofísicos y geohidrológicos. Tesis doctoral. México: Instituto Politécnico Nacional, Centro Interdisciplinario de Ciencias Marinas, 139 pp., 2007.

- GOldMAN, M. y NEUBAUER, F. M. Groundwater exploration using integrated geophysical techniques. Surveys in Geophysics, 15(3): 331-361, 1994.

- JUANAH, M. S. E. et al. Groundwater resources assessment using integrated geophysical techniques in the southwestern region of Peninsular Malaysia. Arabian Journal of Geosciences, 6(11): 4129-4144, 2013.

- KHALIL, M. A. et al. Application of VES and TDEM techniques to investigate sea water intrusion in Sidi Abdel Rahman area, northwestern coast of Egypt. Arabian Journal of Geosciences, 6(8): 3093-3101, 2013.

- LePPER, E. y CURTIS, A. Groundwater development in Lemoa, Guatemala. Environmental Geology, 48(1): 49-56, 2005. 
- MYERS, R. L. Biostratigraphy of the Cardenas formation (Upper Cretaceous) San Luis Potosi, México. Paleontología Mexicana, 24, 1-89, 1968.

- NAVARRO LUNA, R. A. Aplicación de pruebas electromagnéticas en el dominio de tiempo (TDEM) para caracterización de recursos hídricos en Venezuela. Tesis de ingeniería. Venezuela: Universidad Simón Bolívar, 86 pp., 2006.

- OVIEdo garcía, A. Rudistas del Cretácico Superior del centro al sureste de México (recuento sistemático de rudistas americanos). Tesis doctoral. España: Universitat Autónoma de Barcelona, 123 pp., 2005.

- $\quad$ PLATA TORRES, J. L. Técnicas convencionales de geofísica de superficie aplicadas a la hidrogeología. En M. Olmo Alarcón y J. A. López Geta (Eds.), Actualidad de las técnicas geofísicas aplicadas en hidrogeología. España: Instituto Tecnológico Geominero de España, 21-32, 2000.

- QUILANTÁN PEDRAZA, A. et al. Plataforma Valles-San Luis Potosí y la cuenca Mesozoica del centro de México en el Cretácico medio y superior. Boletín de Geociencias, 2(4): 8-14, 2008.

- RAMOS LEAL, J. A. et al. Sucesión de eventos y geometría de la parte central del acuífero del graben de Villa de Reyes (San Luis Potosí, México) inferida a partir de datos geoeléctricos. Revista Mexicana de Ciencias Geológicas, 24(1): 31-46, 2007.

- SAlDiernA, J. J. Análisis hidrogeológico del altiplano de Tula como herramienta de gestión sustentable de recursos hídricos. Tesis de maestría. México: Universidad Autónoma de Tamaulipas, 87 pp., 2014.

- SÁNCHEZ SAN ROMÁN, F. J. Conceptos fundamentales de Hidrogeología. España: Universidad de Salamanca, 11 pp., 2014.

- SÁNCHEZ SAN ROMÁN, F. J. Evolución geoquímica de las aguas subterráneas. España: Universidad de Salamanca, 9 pp., 2012.
- SGM (SERVICIO GEOLÓGICO MEXICANO). Atlas de riesgo de los municipios de Antiguo Morelos, Nuevo Morelos, Ocampo y Tula del estado de Tamaulipas. Reporte. México: Servicio Geológico Mexicano, 256 pp., 2008.

- SGM (SERVICIO GEOLÓGICO MEXICANO). Carta geológicominera estado de Tamaulipas, Escala 1:500,000, 2006.

- TÓtH, J. Groundwater as a geologic agent: An overview of the causes, processes, and manifestations. Hydrogeology Journal, 7(1): 1-14, 1999.

- YOUNG, M. E. et al. Exploration of an alluvial aquifer in Oman by time-domain electromagnetic sounding. Hydrogeology Journal, 6(3): 383-393, 1998.

\section{De páginas electrónicas}

- GOBIERNO DEL ESTAdO DE TAMAULIPAS. Tula [Portal electrónico]. 2011. Recuperado el 6 de marzo de 2015, de http://tamaulipas.gob.mx/tamaulipas/municipios/tula/

- INEGI (INStituto NACIONAL DE EStadísticA, GEOGRAFía E INFORMÁTICA). Información Nacional, por Entidad Federativa y Municipios. 2011. Recuperado el 16 de marzo de 2015, de http://www3.inegi.org.mx/sistemas/mexicocifras/default. aspx?e=28

- KANTA, A. et al. Aquifer characterization using shallow geophysics in the Keritis Basin of Western Crete, Greece. Environmental Earth Sciences, 70(5): 2153-2165, 2013. doi: $10.1007 /$ s12665-013-2503-z

- SIAP (SERVICIO DE INFORMACIÓN AGROALIMENTARIA Y PESQUERA) Cierre de la producción agrícola por estado. s. f. Recuperado el 25 de marzo de 2015, de http://www.siap.gob. $\mathrm{mx} /$ cierre-de-la-produccion-agricola-por-estado/ 